\title{
Fellowship Programs, DCEG
}

National Cancer Institute

\section{Source}

National Cancer Institute. Fellowship Programs, DCEG. NCI Thesaurus. Code C18792.

The National Cancer Institute $(\mathrm{NCl})$ offers training fellowships to individuals who are interested in careers in cancer epidemiology, biostatistics, or genetics. DCEG supports two programs: One provides pre- and postdoctoral training in cancer epidemiology and biostatistics, and the other provides postdoctoral interdisciplinary training in cancer genetics and epidemiology. (from DCEG fellowship programs) 